\title{
Influence of Groundwater Velocity and Surface Temperature on Heat Flow in Aquifers
}

\author{
José Antonio Jiménez-Valera ${ }^{1}$, Gonzalo García-Ros ${ }^{1}$, Iván Alhama ${ }^{1}$ \\ ${ }^{1}$ Technical University of Cartagena \\ Paseo Alfonso XIII, 52, Cartagena, Spain \\ joseantoniojimenez1996@hotmail.com; gonzalo.garcia@upct.es; ivan.alhama@upct.es
}

\begin{abstract}
In this work, it is presented how the heat transfer through a porous medium, with vertical and upward flow of water, is affected when there are changes in the fluid velocity and in the thermal conditions of the ground surface. Using the network method as a numerical tool, these scenarios have been solved, checking how the velocity increase derives in a rise in the heat transfer, although the temperature field is not modified. On the other hand, when the temperature of the soil surface is increased, in addition to rising the heat flow, so do the temperatures of the medium, also developing a transient from the moment of change in the surface thermal condition.
\end{abstract}

Keywords: porous media; groundwater flow; heat transport; surface temperature change.

\section{Introduction}

Heat transport coupled with water flow in porous media is an engineering problem with interesting applications, especially in the field of geothermal $[1,2]$. Its study has involved, among many other contributions, the establishment of a series of benchmark problems, such as those of Bénard, Yusa and Elder [3], which have given rise to numerous scientific publications. In all of them, temperature and velocity fields are coupled, mainly because the flow equation contains a densitydriven term.

For cases where the velocity field is essentially imposed by an external pressure gradient or piezometric levels, a regional flow appears. This simplifies the problem in its mathematical model, although its study and applications are still very useful. In this article, two scenarios in which the flow is predominantly vertical (upwards) are going to be studied, in which the behavior of the heat flow to changes in fluid velocity and ground surface temperature is analyzed. The objective of this study is the quantification of the variation of the heat transmitted through the ground to these changes in velocity and temperature.

For the simulations, a numerical code based on the network method [4] has been used. The free software Ngspice [5] has been used as a calculation tool.

\section{Nomenclature}

$C_{e} \quad$ Specific heat of the porous medium $\left(\mathrm{Jm}^{-3} \mathrm{k}^{-1}\right)$

$D \quad$ Thermal diffusivity $\left(\mathrm{m}^{2} / \mathrm{s}\right)$

$H \quad$ Height or thickness of the porous medium domain (m)

$k \quad$ Thermal conductivity of the porous medium $\left(\mathrm{Jm}^{-1} \mathrm{~s}^{-1} \mathrm{~K}^{-1}\right)$

$K_{x}, K_{y} \quad$ Intrinsic permeabilities of the porous medium in the $\mathrm{X}$ and $\mathrm{Y}$ spatial directions $\left(\mathrm{m}^{2}\right)$

$t \quad$ Time (s)

$T \quad$ Temperature $\left({ }^{\circ} \mathrm{C}\right.$ o K $)$

$V_{0, x}, V_{0, y} \quad$ Water velocity in the in the $\mathrm{X}$ and $\mathrm{Y}$ spatial directions $(\mathrm{m} / \mathrm{s})$

$x, y \quad$ Horizontal and vertical spatial coordinates (m)

$\Delta T \quad$ Temperature at the upper edge of the domain $\left({ }^{\circ} \mathrm{Co} \mathrm{K}\right)$

$\rho \quad$ Density of the porous medium $\left(\mathrm{kgm}^{-3}\right)$ 


\section{The physical and mathematical model}

In general, the equation that governs the flow of heat in a porous medium through which a volume of groundwater circulates, including the phenomena of diffusion and advection, is the following:

$$
\left(\rho C_{e}\right) * \frac{\partial T}{\partial t}-k\left(\frac{\partial^{2} T}{\partial x^{2}}+\frac{\partial^{2} T}{\partial y^{2}}\right)+\left(\rho C_{e}\right) *\left(V_{0, y} \frac{\partial T}{\partial y}\right)+\left(\rho C_{e}\right) *\left(V_{0, x} \frac{\partial T}{\partial x}\right)=0
$$

An equation that, in the absence of horizontal flow, and for a constant vertical velocity $\mathrm{V}_{0, \mathrm{y}}$, is as follows:

$$
\left(\rho C_{e}\right) * \frac{\partial T}{\partial t}-k\left(\frac{\partial^{2} T}{\partial x^{2}}+\frac{\partial^{2} T}{\partial y^{2}}\right)+\left(\rho C_{e}\right) *\left(V_{0, y} \frac{\partial T}{\partial y}\right)=0
$$
below:

Figure 1 shows a physical representation of the scenario. The temperature conditions of the problem are summarized

- Constant temperature at the lower boundary of the domain, of value $\mathrm{T}_{0}=0^{\circ} \mathrm{C}$.

- Constant temperature at the left boundary, of value $\mathrm{T}_{\text {left }}=0^{\circ} \mathrm{C}$, this being the temperature of the fluid at the domain entrance.

- Free temperature at the right edge of the domain.

- $\quad$ Temperature at the upper edge of $\Delta \mathrm{T}$ value.

- $\quad \mathrm{T}_{\text {initial }}$ represents the initial temperature of the water in the medium, with a value of $0^{\circ} \mathrm{C}$.

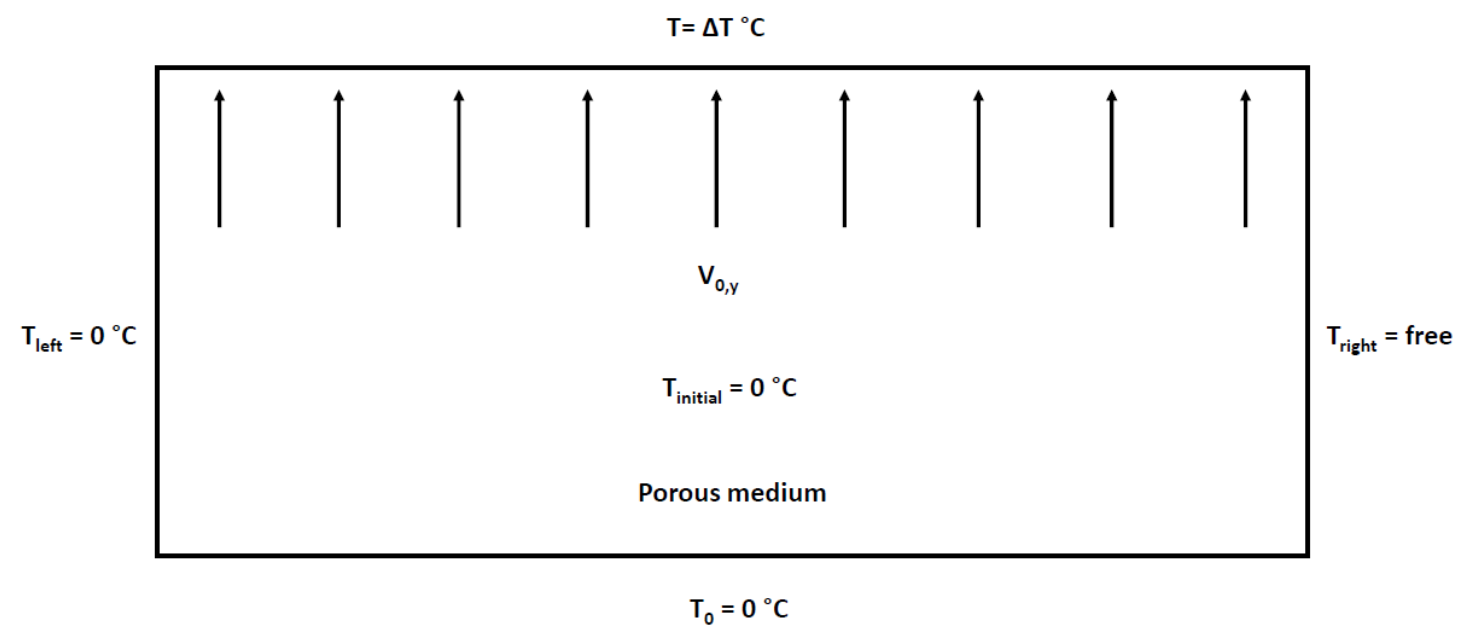

Fig. 1: Temperature conditions of the problem.

Figure 2 shows the geometry of the problem, as well as the values of the intrinsic permeabilities of the soil.

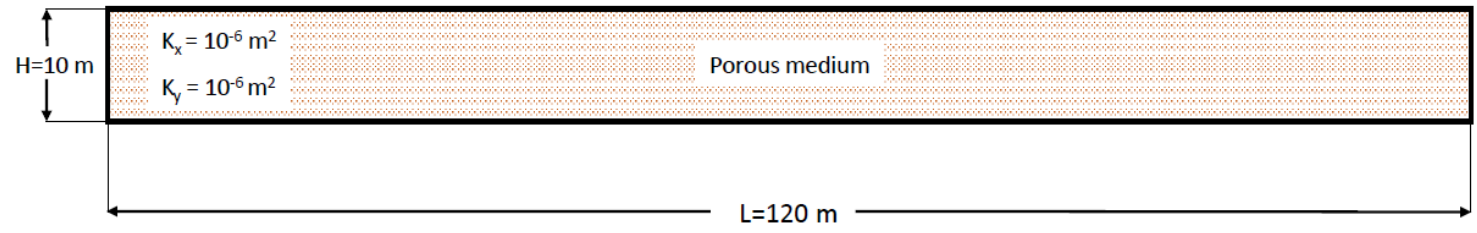

Fig. 2: Geometry of the problem and permeabilities of the soil. 
There are two cases studied here. In the first one, the water enters the lower boundary of the problem with a velocity $\mathrm{V}_{0, \mathrm{y}}=4 * 10^{-6} \mathrm{~m} / \mathrm{s}$, doubling its value once the stationary situation has been reached. In the second case, the water velocity remains constant all the time, with a value $\mathrm{V}_{0, \mathrm{y}}=2 * 10^{-8} \mathrm{~m} / \mathrm{s}$, while the temperature $\Delta \mathrm{T}$ of the upper edge doubles its value once the stationary situation has been reached. The thermal diffusivity $\mathrm{D}=\mathrm{k} /\left(\rho \mathrm{C}_{\mathrm{e}}\right)$ has a constant value of $10^{-5} \mathrm{~m}^{2} / \mathrm{s}$ in both cases.

The governing equation and boundary conditions for this problem are listed below:

$$
\begin{array}{ll}
v(x, y, t)=V_{0, y} & \text { Fluid velocity } \\
\left(\rho C_{e}\right) * \frac{\partial T}{\partial t}-k\left(\frac{\partial^{2} T}{\partial x^{2}}+\frac{\partial^{2} T}{\partial y^{2}}\right)+\left(\rho C_{e}\right) *\left(V_{0, y} \frac{\partial T}{\partial y}\right)=0 & \text { Governing equation } \\
T_{(x=0, y, t)}=T_{\text {left }}=0^{\circ} \mathrm{C} & \text { Left boundary temperature } \\
T_{(x, y=0, t)}=T_{0}=0^{\circ} \mathrm{C} & \text { Lower boundary temperature } \\
T_{(x, y=H, t)}=\Delta T & \text { Ground surface temperature } \\
T_{(x, y, t=0)}=T_{\text {initial }}=0^{\circ} \mathrm{C} & \text { Initial soil temperature }
\end{array}
$$

\section{Simulation and Obtained Results}

The network simulation method [4] has been used as a numerical calculation tool. To solve the equivalent electrical circuits, the free code Ngspice [5] has been used.

\subsection{Case 1: Fluid Velocity Change}

Figures 3 and 4 show the temperatures evolution and the heat transmitted in the medium (at different soil depths), for the case in which we double the velocity $\mathrm{V}_{0, \mathrm{y}}$, passing this from $4^{*} 10^{-6} \mathrm{~m} / \mathrm{s}$ to $8^{*} 10^{-6} \mathrm{~m} / \mathrm{s}$ once the stationary situation has been reached (100 Ms).

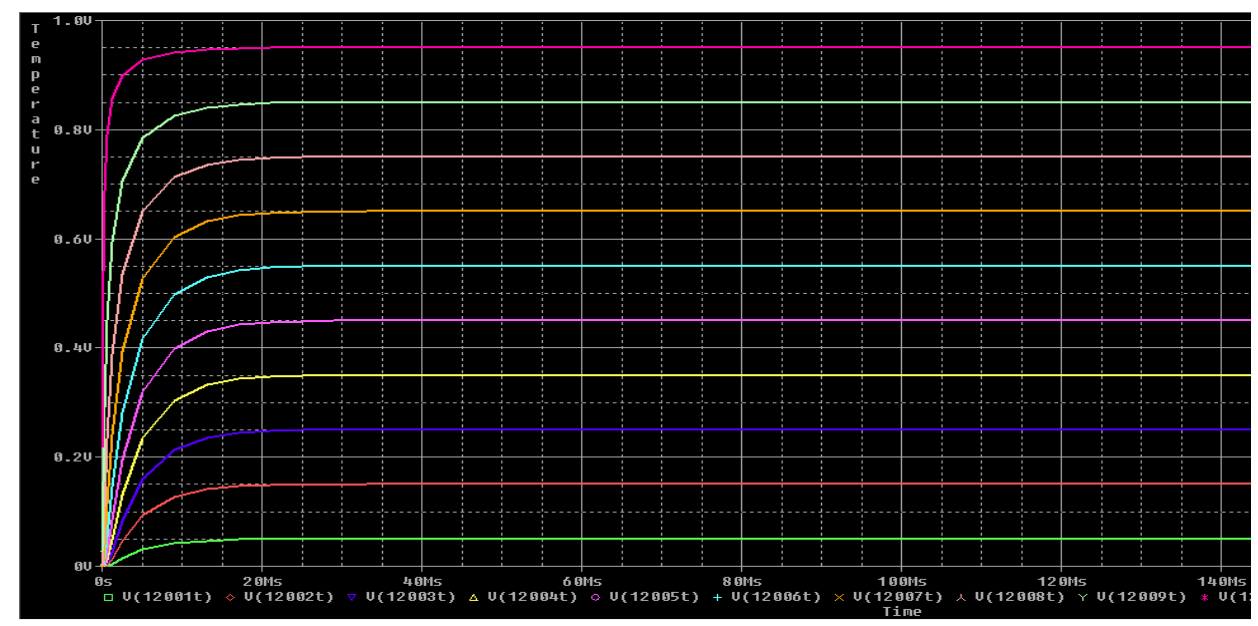

Fig. 3: Evolution of temperatures over time for case 1.

As can be seen, the temperatures remain constant when the velocity changes (Figure 3), while the transmitted heat does double in value (Figure 4). The increase in this last variable is observed linear and progressive since the change in fluid velocity has been implemented in this way. That is, no transitory phenomenon generated by the velocity change is observed. 


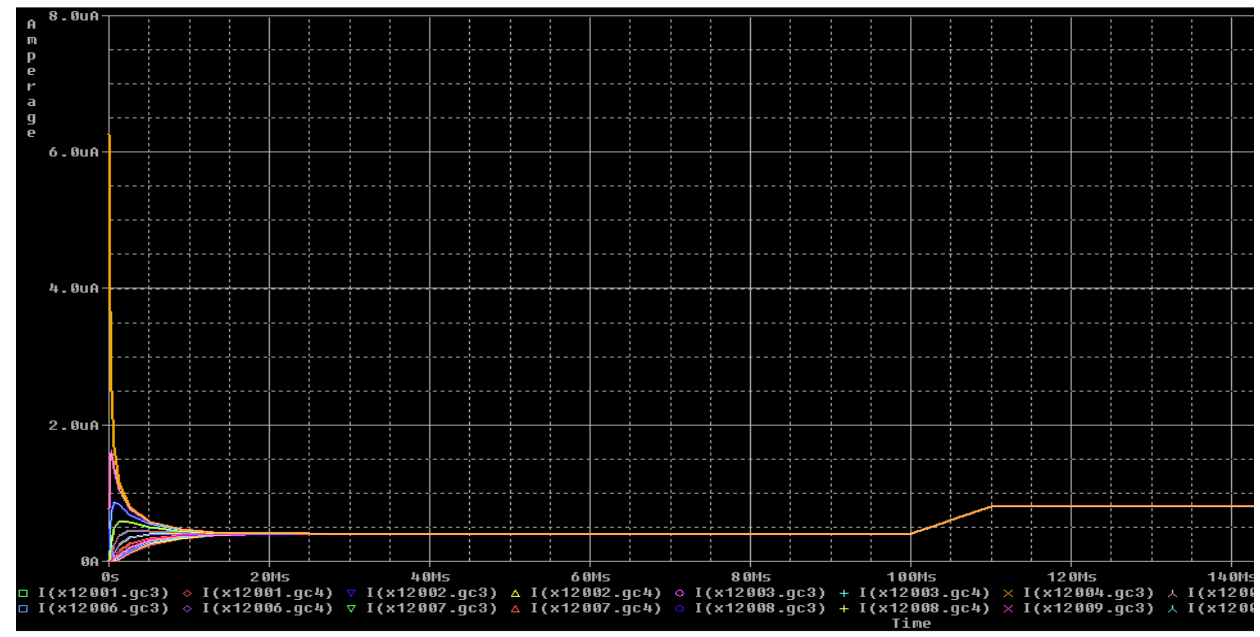

Fig. 4: Evolution of the heat transmitted over time for case 1.

\subsection{Case 2: Change in Ground Temperature}

Figures 5 and 6 show the temperatures evolution and the heat transmitted in the medium (at different soil depths) for the case in which we double the ground surface temperature $\Delta \mathrm{T}$, going from $1^{\circ} \mathrm{C}$ to $2^{\circ} \mathrm{C}$ once the stationary situation has been reached (100 Ms).

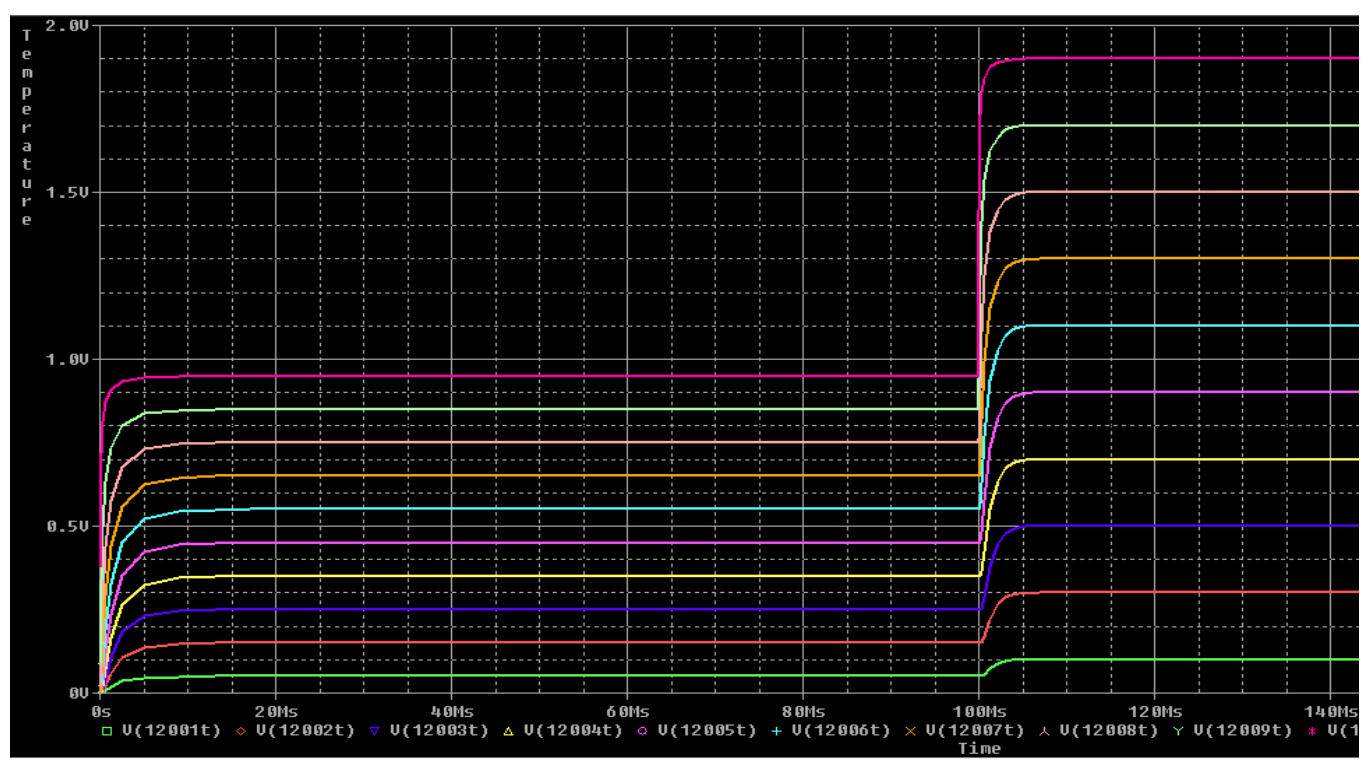

Fig. 5: Evolution of temperatures over time for case 2.

As can be seen, both temperatures (Figure 5) and transmitted heat (Figure 6) double as the surface temperature does. In this case, although the temperature change is immediate, the generation of a second transitory phenomenon is observed. 


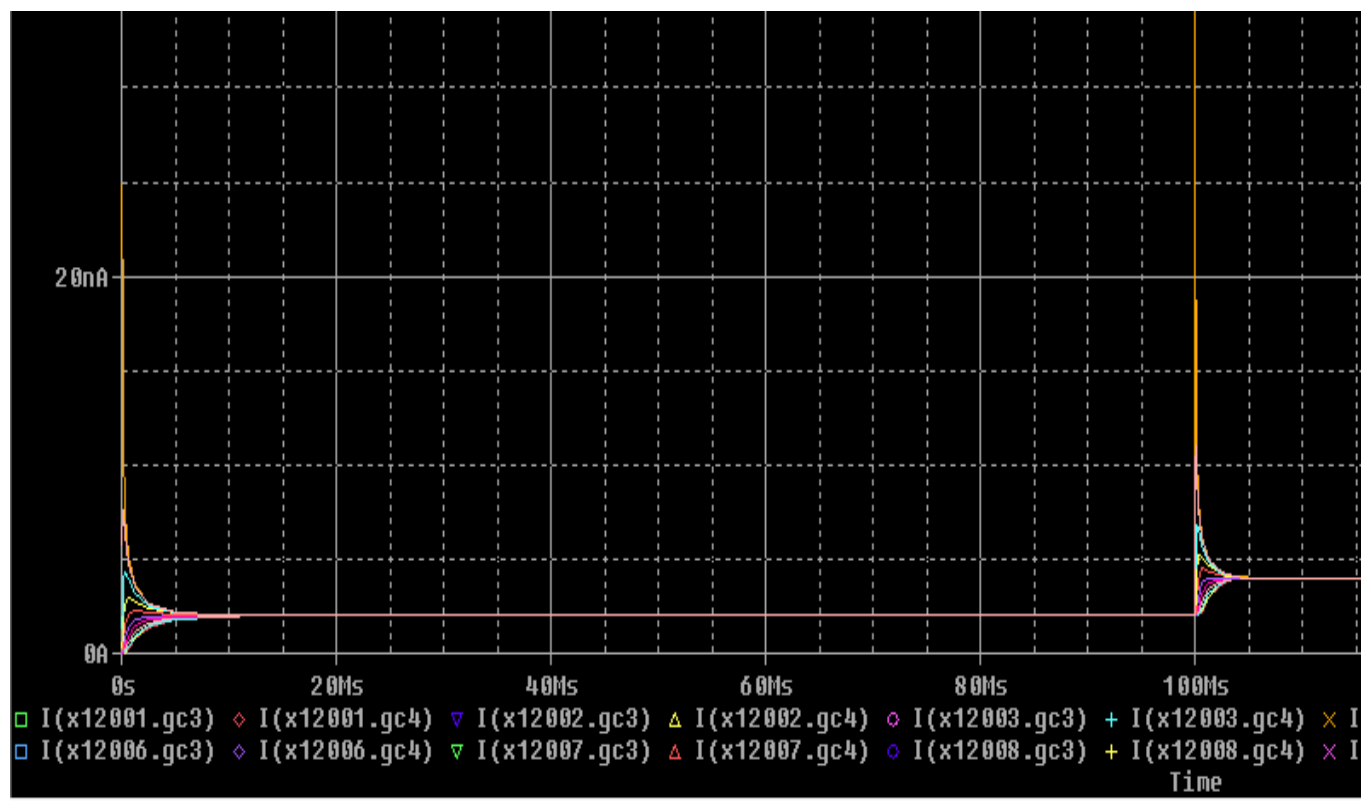

Fig. 6: Evolution of the heat transmitted over time for case 2.

\section{Conclusion}

The simulations carried out have made it possible to verify that, in the event of an increase in the fluid velocity, the temperature field is not affected. However, changes in the transmitted heat are observed, although in no case the appearance of transient phenomena is observed.

On the other hand, when what is increased is the temperature of the ground surface, in addition to increasing the heat flow so do the soil temperatures, also observing the appearance of a transient from the moment in which the temperature is changed.

\section{References}

[1] E. Di Sipio, S. Chiesa, E. Destro, A. Galgaro, A. Giaretta, G. Gola and A. Manzella. "Rock thermal conductivity as key parameter for geothermal numerical models." Energy Procedia, vol. 40, pp. 87-94, 2013.

[2] M. Barla, A.D. Donna and M. Baralis. "City-scale analysis of subsoil thermal conditions due to geothermal exploitation." Environmental Geotechnics, pp. 1-11, 2018.

[3] M. Cánovas. Caracterización adimensional y simulación numérica de escenarios patrón (Bénard, Yusa y Elder) de procesos geotérmicos de flujo y transporte. Doctoral dissertation. Universidad Politécnica de Cartagena, 2014.

[4] C.F. González-Fernández. Applications of the network simulation method to transport processes, in Network Simulation Method. Ed. J. Horno, Research Signpost, Trivandrum, India, 2002.

[5] Ngspice - Open Source mixed mode, mixed level circuit simulator (based on Berkeley's Spice3f5), 2016. Available: http://ngspice.sourceforge.net/ 